\title{
Profiling and Assessment within Case Studies
}

The profiling and assessment strategy adopted by the Joint Unit within the Foundation Programmes is based on a structure of reviewing each Case Study on its completion and setting goals for the future as a result of that review. A differentiated assessment statement may be produced for each of the Case Studies, indicating both the experience that the students have undertaken and the level of skills that was demonstrated and assessed during that experience. Completion of any assessment documents takes place in the classroom as an integral part of the Case Study itself.

The differentiation in a student's performance will be indicated through context as well as skill. For example, the making of a cup of tea for some students may be quite an achievement whereas other students may be using complex video subtitling equipment and programming computers. It is therefore not only the level of skill that will give an indication of performance but also the statement of the activities themselves.

Details of the profiling strategy and reporting procedures are contained in the Joint Unit publications Profiling Procedures, Profiling Handbook and The Plain Person's Guide to Profiling - all are available from City and Guilds or BTEC. The method of setting assessment objectives for a unit of work and recording achievement against those objectives is also a common strategy for subject-based assessment and the general strategy of recording and reflecting on a unit of work - in this case a Case Study and setting targets for the future is the methodology promoted by the Record of Achievement and Profiling Pilots. As a methodology therefore the Joint Unit strategy can be developed across the whole curriculum, as a strategy for the assessment of cross-curricular skills.

\section{DOCUMENTATION}

As presented by the Joint Unit in their Profiling Procedures publication, the Case Study Review sheet is the primary tool for ongoing formative assessment and the Assessment Statement is the method of reporting on achievement, and is therefore the interim tool for summative reporting.

\section{Review Sheets}

The review sheets can be designed by the school to reflect a 'house style', and to fit in with the profiling system, they can be designed to meet the needs of an individual Case Study or the school can use the standard Joint Unit example provided on page 308 . Because of this variation, review sheets are not generally provided as part of the Case Studies within this pack.

\section{Assessment Statements}

The Assessment Statements were designed for summative reporting although the Joint Unit has recently relaxed its requirements for these to be produced to the Formal specification. Our authors have interpreted the original specification slightly differently and we have made no attempt to standardise them although we have included them at the end of the Student Task Sheets for teachers to use if they see fit. Attempts have been made in Case Study 13 to emphasise content and to minimise differentiation, in Case Study 8 attempts have been made to produce a single coherent paragraph, and in Case Study 5 to identify the skills in relation to tasks and to code numbers. All of these examples meet the specification and can translate directly into the final profile accredited by the Joint Unit. It must be borne in mind however that students may only select some of these statements for final reporting purposes and that alternative methods of recording differentiated skills in context may be used during the programme if they were felt to be more appropriate.

Within the Foundation Programmes the content is indicated by a description of the task and for each task one or two skills are chosen for assessment purposes. The skills chosen by the authors for assessment within each of the Case Studies in this pack are shown in figure 3.

The new, more flexible, Joint Unit requirements for profiling and final reporting are detailed in The Plain Person's Guide to Profiling - available from City and Guilds or BTEC. 